- Fermilab

$\overline{\mathrm{p}}$ Note \#382

SOME SUGGESTED TECHNIQUES FOR ACHIEVING REQUIRED MAIN RING LOW LEVEL RF PERFORMANCE FOR THE TEVATRON I

PROGRAM

J.E. Griffin \& J. MacLachlan

May, 1984 
$\bar{p}$ Note 382

J.E. GriffinJ. MacLachlan

May 1984

\section{Some Suggested Techniques for Achieving Required Main Ring Low Level RF Performance for the Tevatron I Program}

These techniques are derived from experience with the existing low level rf system and from the results of accelerator experiments directed at the Tev I requirements which were performed using a specially modified low level rf system. The techniques suggested here are not necessarily unique and other techniques may be acceptable.

It is important to realize that certain performance requirements on the Booster and Main Ring are substantially different in the Tev I program from those which have been acceptable in the fixed target program. For example, the longitudinal enittance of the bunches has been routinely and intentionally blown up by the "bunch spreader" during acceleration to improve the smoothness of the spill. In the Tev I case, the "bunch narrowing" procedure preceding $\overline{\mathrm{p}}$ production and in bunch coalescing is facilitated by maintalning the smallest possible longitudinal enittance (i.e. the largest possible longltudinal phase space density). Also, during fixed target physics the Main Ring is essentially full (1.e. approx. 1090 of 1113 buckets occupled) so transient beam loading of the of system is negligible except during injection, whereas for $\bar{p}$ production a single high intensity Booster batch (approx. 83 bunches) is to be accelerated causing substantial transient beam Induced phase shift of the rf voltage during each passage. The low level phase and position error signals are normally derived from an essentially full ring during fixed target operation whereas in the Tev I program the systems must perform adequately with as few as ten adjacent buckets occupied.

Because of the differences in emphasis between fixed target operation and Tevatron I operation is seems sensible to use separate low level $\mathrm{rf}$ systems for these two modes of operation. If a single low level system were to be used for both modes of operation some compromises may be necessary which might cause performance in elther mode to be less than adequate. Because of the severe demands made on accelerator performance in the Tev I program it would seem reasonable to develop a unique system which can be optimized to the requirements.

1. Longitudinal Dilution at Injection

In normal operation the Main Ring $r f$ frequency is held fixed during thirteen Booster acceleration cycles and the Booster $\mathrm{rf}$ is phase-locked to the fixed frequency during the last $3-5 \mathrm{msec}$ of each cycle. These Booster phase-locks cause a variable amount of dilution, depending upon somewhat random initial conditions, on each cycle. This procedure is necessary because of the succession of thirteen Booster batches required to fill the 
Maln Bing. In the Tev I program only one (or less than one) Booster batch is accelerated on each cycle so the procedure can be reversed; the Main Ring frequency and phase can be locked to the Booster near the end of the Booster cycle. This will eliminate the rapld and somewhat varlable bucket motion just prior to Booster extraction. It should be possible to adjust the phase and amplitude of the Main Ring buckets so that the injected bunches are accurately matched to the buckets. At the time of Booster extraction the Main Ring-Booster phase-lock should be terminated and the Main Ring frequency and phase held flxed until well established beam induced feedback signals can be applied.

The same procedure should be applied when antiprotons are injected from the Accumulator ring except that the Main Ring should then be locked to the Accumulator $\mathrm{rf}$.

It seems reasonable to use the same phase detector to accomplish these two functions with an $r f$ switch to select the rf source to which the system is locked. An additional switch position could select an rf signal derived from beam in the Main Ring at an appropriate time. With an appropriate transfer function the transient disturbance delivered to the VCO when $r f$ sources are switched could be minimized.

An even simpler scheme, involving no switches is shown in Figure 1. An amplified and gain-controlled signal derived from the Booster beam is delivered to a combiner, the output of which is delivered to the phase detector and compared to the delayed MRRF VCO output (such a signal exists in the Booster low level rf system). In this way the Main Ring rf is locked to the Booster beam just prior to injection. A slgnal from the Main Ring beam pickup, suitably amplified and leveled, is delivered to another Input of the combiner. When beam 1s extracted from the Booster the Booster signal disappears and a few microseconds later a signal appears from the Main Ring pickup. With phases properly adjusted a smooth transfer of phase lock with minlmum transient disturbance should occur automatically.

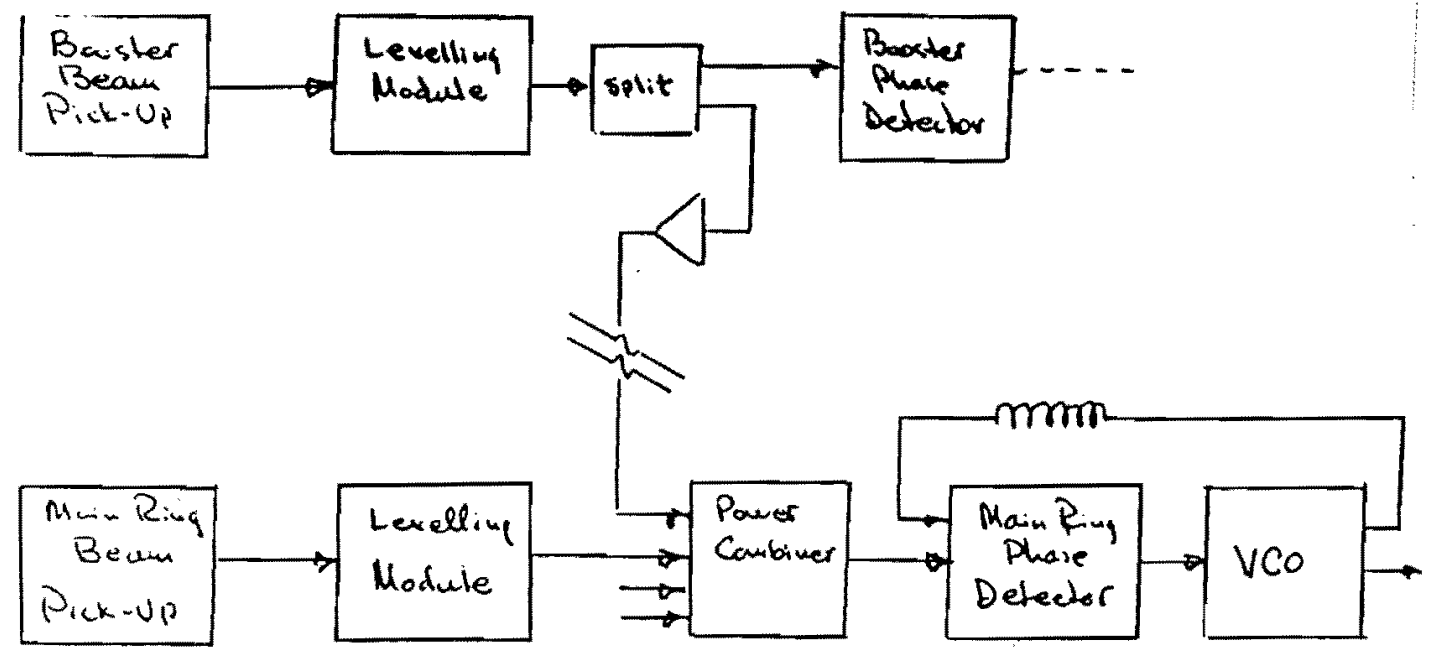


Signals from other sources, such as the Antiproton Accumulator Ring can be delivered to the combiner in a similar way. There may be additional technical complexity relating to the number of bunches involved or unwanted signals so some additional gating may be necessary.

Alternatively, several phase detectors, each dedicated to a particular function, could be employed. It would seem that the likelihood of transient disturbance of the VCO at switching times would be worse in this configuration but it may be possible to avoid this problem in some way. This choice would appear to require significantly more bin space than using rf switching. If more than one or two phase detectors are to be switched into the VCO the problem of the of fset voltages between units may become difficult to manage.

\section{Small Ensemble Acceleration}

In the acceleration of an ensemble of a few bunches (approx. 10) or a single Booster batch it is important that the error signal from the phase detector delivered to the VCO remain at an acceptably stable and constant value during those periods (approx. 20 microseconds each turn) when beam signals are absent. A simple approach to the problem is to use a Track and Hold element between the phase detector and the VCO.

Unfortunately this technique is particularly difficult when the T\&H system is used to deliver an error signal in a feedback loop, as is required here, because the error signal is nominally zero and the T\&H offset voltage or "pedestal" may be comparable in magnitude to a significant error voltage. One way to manage this problem is to amplify the error signal as much as is practical ahead of the T\&H and to follow the T\&H with equivalent attenuation. Since the pedestal is constant its effect is reduced by the attenuation. Another approach is to digitize the error signal and deliver updating digital information to a digitally controlled variable frequency oscillator. "Hold" is accomplished by terminating the updating process.

Suppose, as an example, that the "track to hold" of fset voltage is 25 $\mathrm{mV}$. If the VCO has a frequency to voltage sensitivity of $50 \mathrm{kHz}$ per volt the offset will result in a frequency shift of $1250 \mathrm{~Hz}$ or an angular frequency of $2500 \pi$ radians per second. In the $19.3 \mathrm{microsecond} \mathrm{period}$ between passage of single batches this frequency shift will result in a phase shift (compared to the "correct" frequency with no frequency shift) of about $0.05 \pi$ radians or 9 degrees. A phase shift of this magnitude in the bucket location with respect to the returning beam would be a matter of concern. Fortunately, the rf cavities act as a flywheel and they cannot respond fully to this drive phase change in one rotation period. Nevertheless such a phase shift is not negligible and some consideration should be given to reducing or eliminating 1 t.

The existing radial position modules have a gating feature which is ideally suited to operation of a small ensemble T\&H unit. This signal is 
called the "mode" signal. The signal is shown in Figure 2 along with the rf output of the leveling module. The signal starts in the high or "track" condition, goes low at the end of ensemble passage, then goes high on each successive passage. Because of the relative location of the pickups involved the relative times of the signals are 1deal. Unfortunately this time relationship will not be preserved for antiprotons going the other direction so some other source of gating information may be required.
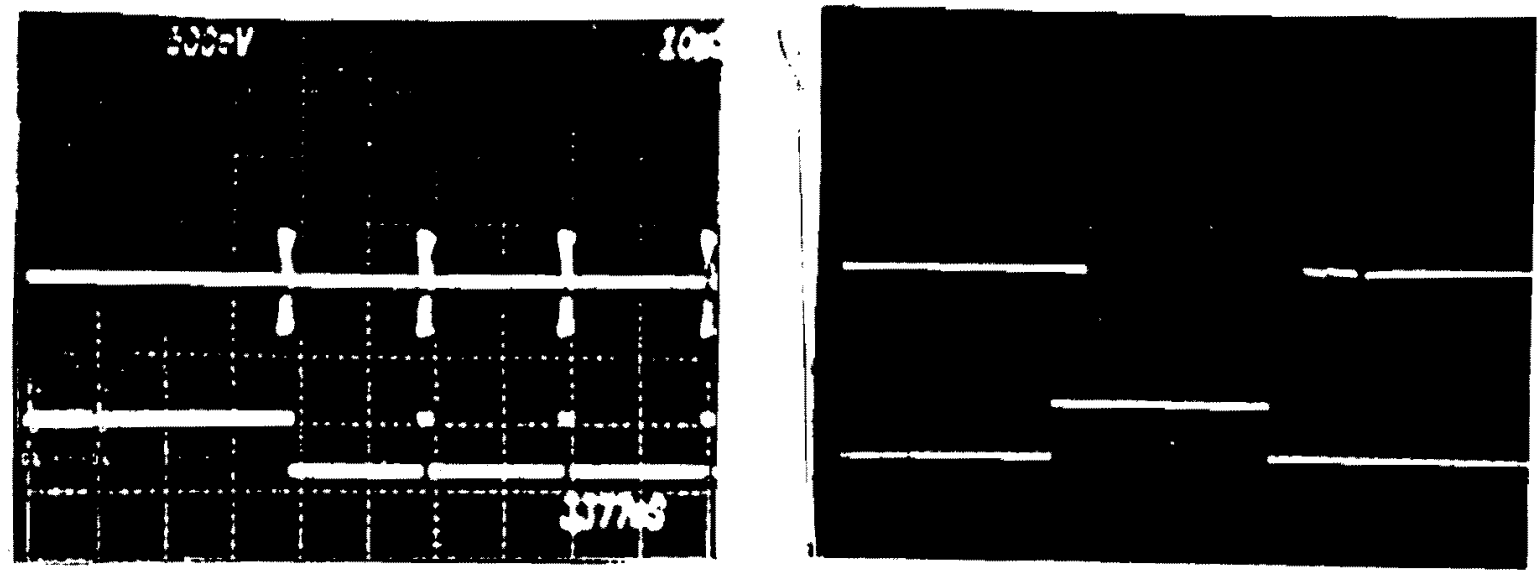

Figure 2

"Mode" signal from radial position system, with beam signal from leveling module.

\section{Translent Beam Loading}

A single Booster batch with 84 bunches each containing $\mathrm{g} 2.5 \times 10^{10}$ protons will induce a voltage in the Main Ring rf system varying from zero to about $100 \mathrm{kV}$ during a $1.6 \mathrm{mlcrosecond} \mathrm{passage.} \mathrm{This} \mathrm{transient} \mathrm{voltage}$ will decay to about half this value during the $19.3 \mathrm{microseconds}$ while the batch is elsewhere and recur at each batch passage of the rf cavities. In a stationary bucket situation with nominally $10^{6}$ volts developing buckets, this transient voltage will cause a phase shift of about 6 degrees. This means that some bunches in the batch will be mismatched to the bucket center and the ensuing coherent dipole motion will cause a longitudinal density dilution. Figure 3 shows two traces of the phase shift resulting from passage of a single batch (approx. 84 bunches) of approx. the intensity mentioned above through the Main Ring of system at $8 \mathrm{Gev}$ while the system was developing about $1 \mathrm{MV}$. The two traces were separated by adding a length of line corresponding to 7.2 degrees of phase shift. The transient beam loading phase shift is about 5 degrees. 


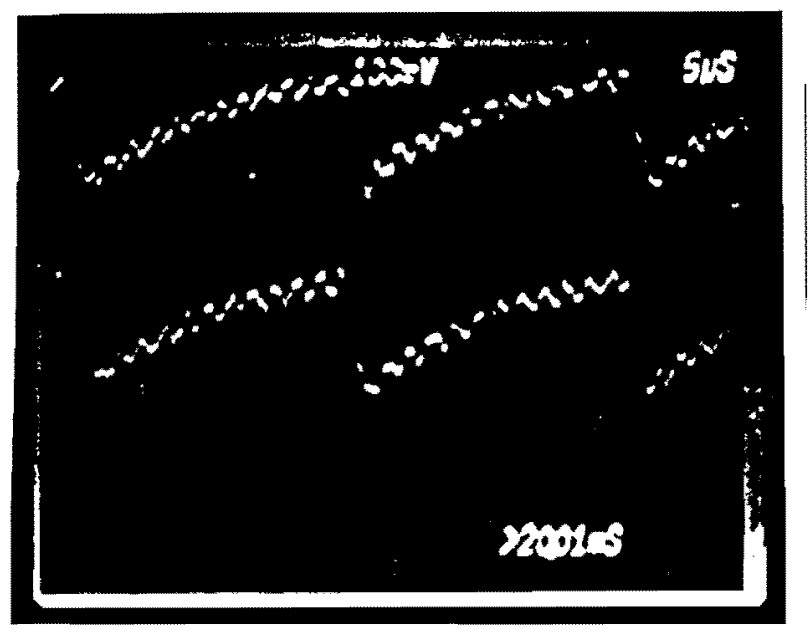

Figure 3

Phase Shift in the Main Ring of voltage resulting from passage of a single Booster batch of beam through the Main Ring $r f$ system at $8 \mathrm{GeV}$.

During acceleration the transient voltage will cause a variation in bucket area over the batch in addition to the phase shift.

In principle the transient beam loading could be eliminated by deliverying to the $\mathrm{rf}$ cavities a burst of current equal and opposite in time and amplitude to the $53 \mathrm{MHz}$ Fourier component of the passing beam bunches. There is no simple way to do this directly although one could think of pickups located part way around the ring and direct cables to Fo. A simple solution would be to sample the beam passage at FO, remember the information for one turn (about 20 microseconds) and introduce compensating current derived from the information into the $\mathrm{rf}$ system always one turn late. Unfortunately there is a large quantity of information contained in 84 bunches and there are technical difficulties associated with remembering accurately the information for $20 \mathrm{microseconds}$ and returning it to the system. Since the cavities respond only to the $53 \mathrm{MHz}$ component of the beam current (we suppose), it is necessary to remember only the amplitude, frequency, and phase of this component. The held VCO output frequency does part of this job, remembering the frequency and phase, subject to the $T \& H$ pedestal and drift errors mentioned earlier. To a large extent the transient beam loading can be compensated for in the low level rf system by feeding a burst of the VCO output signal around the operational phase shifters (including counter-phase modules) and adding it to the fan-out voltage. The signal must be gated to the $\mathrm{rf}$ cavities only when the beam is passing and its amplitude must be adjusted to the beam intensity. An appropriately timed gating signal can be developed using the $47 \mathrm{kHz}$ rotation frequency clock and an adjustable delay. The amplitude of the signal fed forward should be adjusted automatically to match the beam 
Intensity on each acceleration cycle. The VCO output $\mathrm{rf}$ is alway phase-locked to the beam and fixed phase shifts are Introduced such that when the operational phase shifter is not driven (1.e. Input voltage is zero, corresponding to zero relative phase shift) the rf cavities develop a stationary bucket. At injection, below transition, the beam current leads the $\mathrm{rf}$ gap voltage in phase by $\pi / 2$ radians. If the fed forward voltage is to excite the rf cavities at a phase opposite to the beam excitation then the phase must lag the VCO voltage delivered to the operational phase shifter by an additional $\pi / 2$ radians.

In Figure 4 the fan-out fan-back phase shift with and without compensation is shown for a single Booster batch. Figure $5 \mathrm{a}$ shows the relative gating time in relation to the beam signal from the leveling module at the low level rf bin location. Flgure $5 b$ shows the fan-out rf signal during this compensation. During this experiment application of the compensation caused a repeatable 13 percent increase in beam intensity at $100 \mathrm{GeV}$ for normalized injection conditions.

\section{FIGURE 4}

Improvement in fan-out fan-back beam induced phase shift with feed-forward compensation.

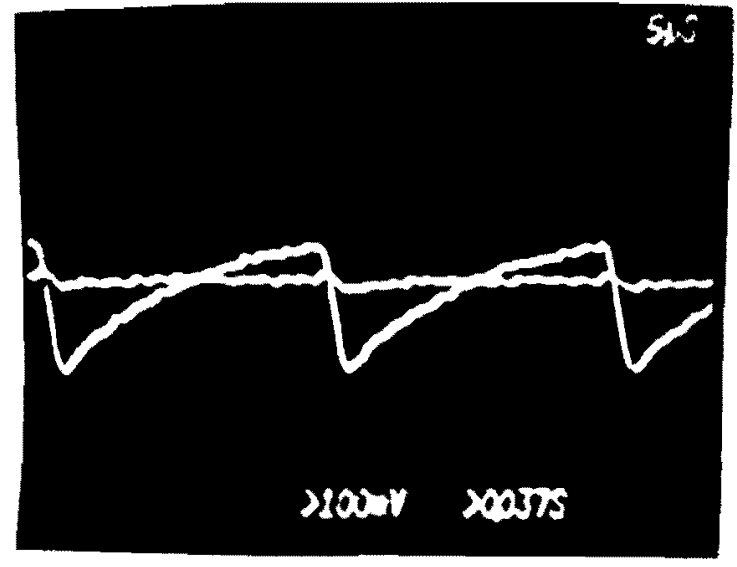

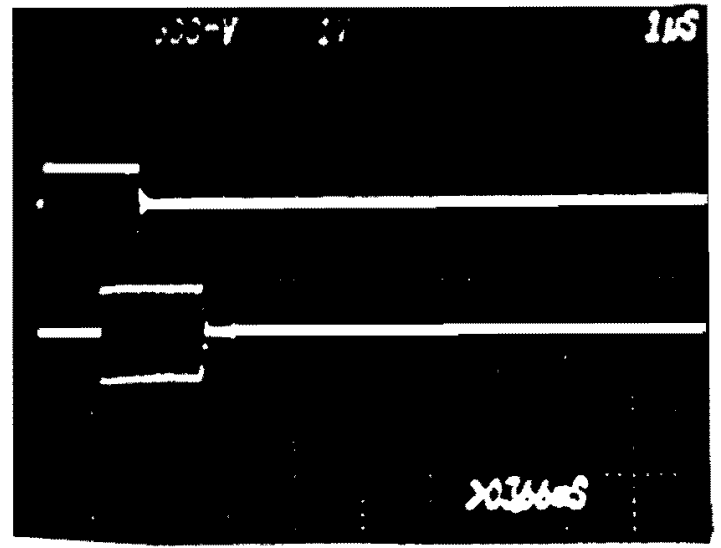

(a)

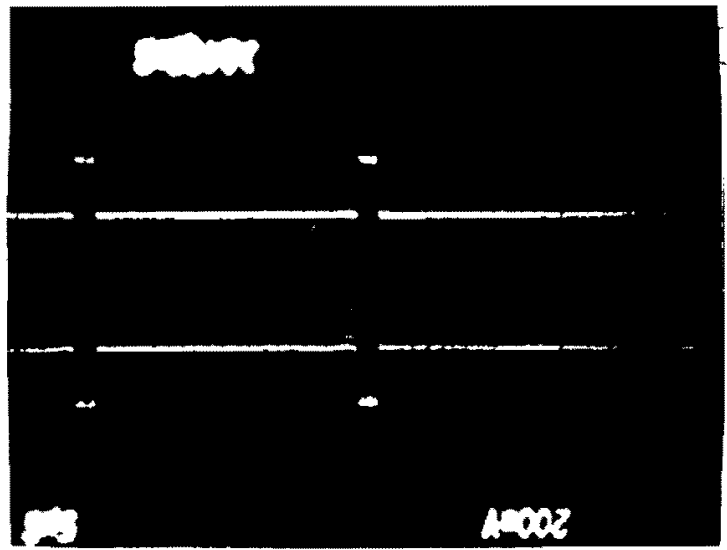

(b)

Figure 5

(a) Optimum gating timing for compensation with respect to beam signal from leveling module. (b) Fan-out rf.

The increase in beam intensity presumably resulted from improved passage through transition of bunches with reduced longitudinal dilution. 
The procedure outlined above for transient beam loading compensation w1ll probably work adequately for single Booster batch acceleration. Bunch lengths during these operations remain reasonably constant and the $53 \mathrm{MHz}$ component of the distributions remains reasonably constant. Several problems may arise during flattop operations. First the $\mathrm{rf}$ is to be derived from a flattop oscillator rather than from the VCO and some problems may arise in feeding the VCO signal forward and adding it to the fan-out rf. In princlple this should work as before because the vco should be phase-locked to the beam regardless of the actual source of $\mathrm{rf}$. In practice this phase-lock may not be adequate for some unexpected reason.

The second problem arises during rf voltage reduction for bunch broadening. For a fixed intensity the $53 \mathrm{MHz}$ Fourler component of beam exclting the rf system will decrease as the bunches are broadened. While this decrease may only be a factor of about 0.75 it may st1ll cause a compensation error just when the required resultant voltage is decreasing to an amplitude which is, in fact, smaller than the beam induced voltage. A Booster batch with $2 \times 10^{10}$ protons per bunch and $S=0.3 \mathrm{eV}-\mathrm{sec}$ per bunch (design study values) can induce a maximum voltage of about $45 \mathrm{kV}$ in the system while the required voltage is about $34 \mathrm{kV}$. If the anode program and cascode current on each $\mathrm{rf}$ cavity is reduced to too low a value during counter-phasing, there may not be enough rf amplifier current avallable to compensate for beam loading even if the fed-forward signal is at the "correct" value.

Two examples of counter-phased $r f$ voltage with improperly adjusted compensation and one example of approximately correct compensation are shown in Figure 6 . 

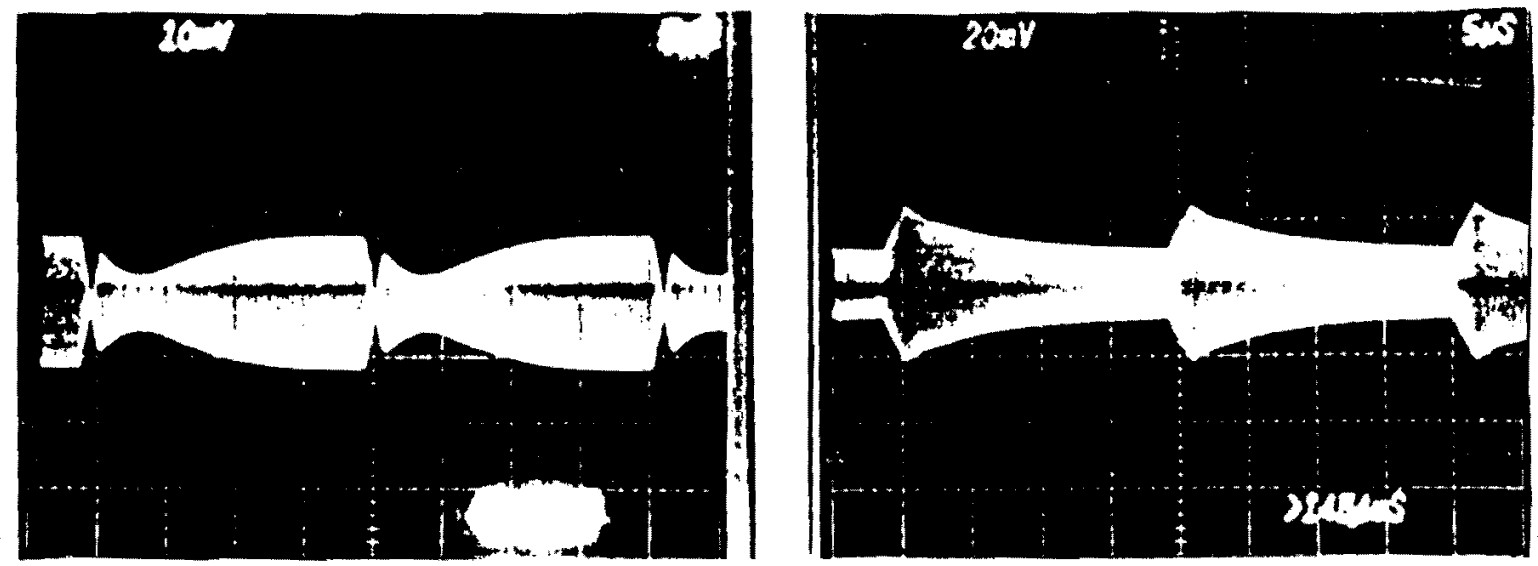

(a)

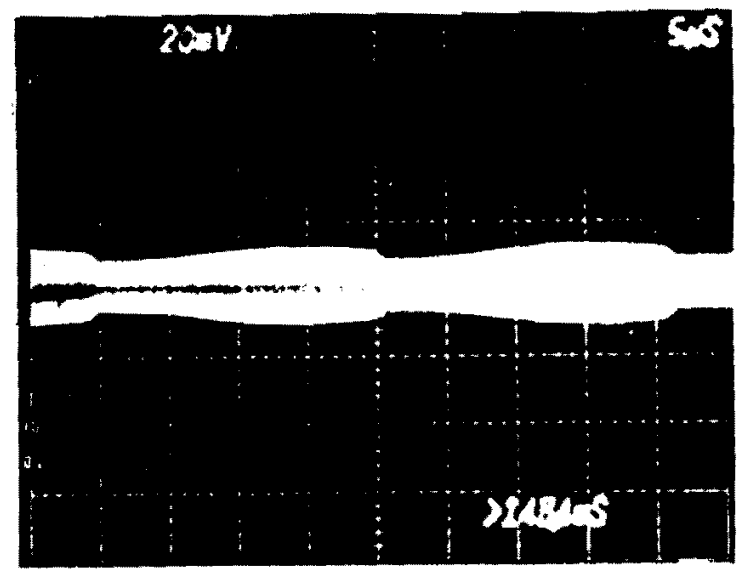

(b)

\section{Figure 6}

(a) Improperly adjusted translent beam loading of voltage during counter-phasing. (b) Approximately correct adjustment of transient beam loading compensation during rf counter-phasing.

Translent beam loading from small ensembles during adiabatic debunching prior to and during coalescing at $h=53$, may present a more difficult problem. The "debunched" small ensembles may, in some configurations, develop voltages in the rf system which are comparable to or larger than those required to debunch the beam. These voltages may be developed even when the MR cavities are of $f$ during bunch rotation at $h=53$. This situation begins to look like an instability rather than a standard beam loading problem, and a separate document treating the problem is being prepared.

4. RF Voltage Reduction on Flattop

During $\bar{p}$ production and during the bunch coalescing process necessary to produce intense $p$ and $\bar{p}$ bunches the $r f$ voltage must be reduced to very 
small values during the $120 \mathrm{GeV}$ and $150 \mathrm{GeV}$ flattops. It seems reasonable to accomplish this reduction by counter-phasing approximately equal numbers of $\mathrm{rf}$ cavities while at the same time reducing the voltage developed by each cavity to the smallest possible value. At least one and probably two feedback loops (on phase and amplitude) will be required to control the amplitude and maintain the proper phase of the reduced bucket.

In the $\bar{p}$ production mode the net $\mathrm{rf}$ voltage creating stationary buckets must be reduced to the order of $10 \mathrm{kV}$ before it is raised to its maximum value (about $4 \mathrm{MV}$ ). About $100 \mathrm{msec}$ w1ll be required for this reduction and the voltage profile is dictated by the requirement of adlabaticity and minimum dilution. At the end of the voltage reduction the bunch should extend over a range $\pm \pi / 2$ radians of $\mathrm{rf}$ phase. In order to reduce the bucket area smoothly with a minimum of dilution, the incremental change in bucket area, $d S$, should be adjusted to be a constant fraction of the phase oscillation period $\tau$. This condition can be described by an adiabaticity parameter $\alpha$,

$$
\alpha=\text { const. }=\frac{\tau}{S} \frac{d S}{d t}
$$

The ratio $\alpha$ should be small for small dilution but empirically it is found that 1 t can be as large as 2 without serlous dilution. The resulting voltage program can be expressed

$$
V(t)=\frac{1}{\left(1-\frac{\alpha t}{\tau}\right)^{2}}
$$

where, for a process taking $\mathrm{N}$ turns

$$
\alpha=\left[1-\left(\mathrm{V}_{\mathrm{O}} / \mathrm{V}_{\mathrm{N}}\right)^{1 / 2}\right] \frac{\mathrm{T}_{\mathrm{O}}}{\mathrm{NT}}
$$

where: $V_{0}=r f$ voltage at start

$\mathrm{V}_{\mathrm{N}}^{\mathrm{O}}=\mathrm{rf}$ voltage after $\mathrm{N}$ turns

$\mathrm{T}_{0}^{\mathrm{N}}=$ rotation period of Main Ring

$\tau_{0}^{0}=$ synchrotron phase oscillation period at start.

In the design study case, $2 \times 10^{10}$ protons per bunch, each with $S=0.3$ $\mathrm{eV}-\mathrm{sec}$ the final value of the $\mathrm{rf}$ voltage required to spread the bunches over $\pm \pi / 2$ radians is about $35 \mathrm{kV}$. The phase osclilation period at $1 \mathrm{MV}$ and 
$120 \mathrm{GeV}$ is $0.01 \mathrm{sec}$ so an adiabaticlty factor of $\alpha$ of -1 results in a debunching period of about two thousand turns or about $42 \mathrm{msec}$. The time dependence of the $\mathrm{rf}$ amplitude is

$$
V(t)=\frac{1}{(1+100 t)^{2}} M V
$$

The expression for $\alpha$ shows that it is only weakly dependent on $V_{0}$ if $V \gg V_{N}$. Therefore 1 matters little whether the starting voltage is $1{ }^{\circ} \mathrm{MV}$ or as high as $4 \mathrm{MV}$. However, if an attempt has been made to minimize dilution during the final phase of acceleration by keeping the bunch length nearly constant during the fleld parabola from acceleration to flattop, the rf voltage will have been reduced to about $1 \mathrm{MV}$, as used in the above example.

A plot of a voltage reduction curve with adiabaticity factor -0.5 is shown in Figure 7. The curve starts at $1 \mathrm{MV}$ and $1 \mathrm{t}$ is followed by a fast excursion to $4 \mathrm{MV}$. 


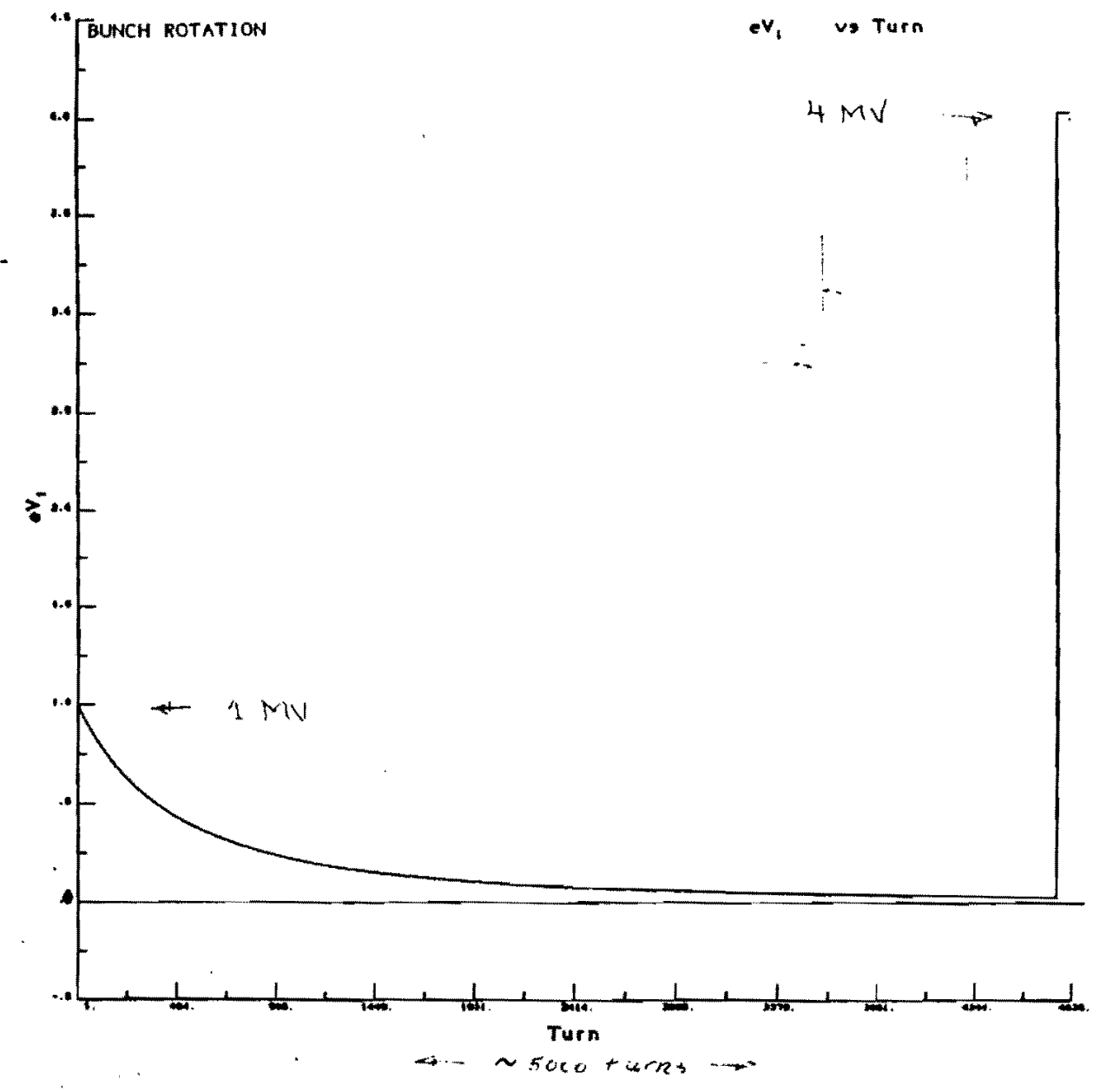

F1gure 7

Adiabatic $r f$ voltage reduction curve for $\bar{p}$ production.

At the end of the voltage reduction phase the voltage must be raised as quickly as possible to the maximum possible value and held at that level for one quarter of a synchrotron period. At $4 \mathrm{MV}$ and $120 \mathrm{GeV}$ the synchrotron period is about $5 \mathrm{msec}$ so the beam will be extracted about 1.3 msec after the voltage is raised.

In feasibility experiments bunch lengths of 1 nsec full width were obtained using the above described procedure and a maximum rf voltage of $3.5 \mathrm{MV}$. The results indicated that the longltudinal enittance of the bunches in the experiment were about $0.3 \mathrm{eV}-\mathrm{sec}$ and a counter-phase rf voltage of about $26 \mathrm{kV}$ was sufficient to spread the bunches over half the bucket length. In Figure $8 \mathrm{a}$ and $8 \mathrm{~b}$ the $\mathrm{rf}$ voltage reduction profile and the resulting bunch shape are shown prior to sudden raising of the voltage. 

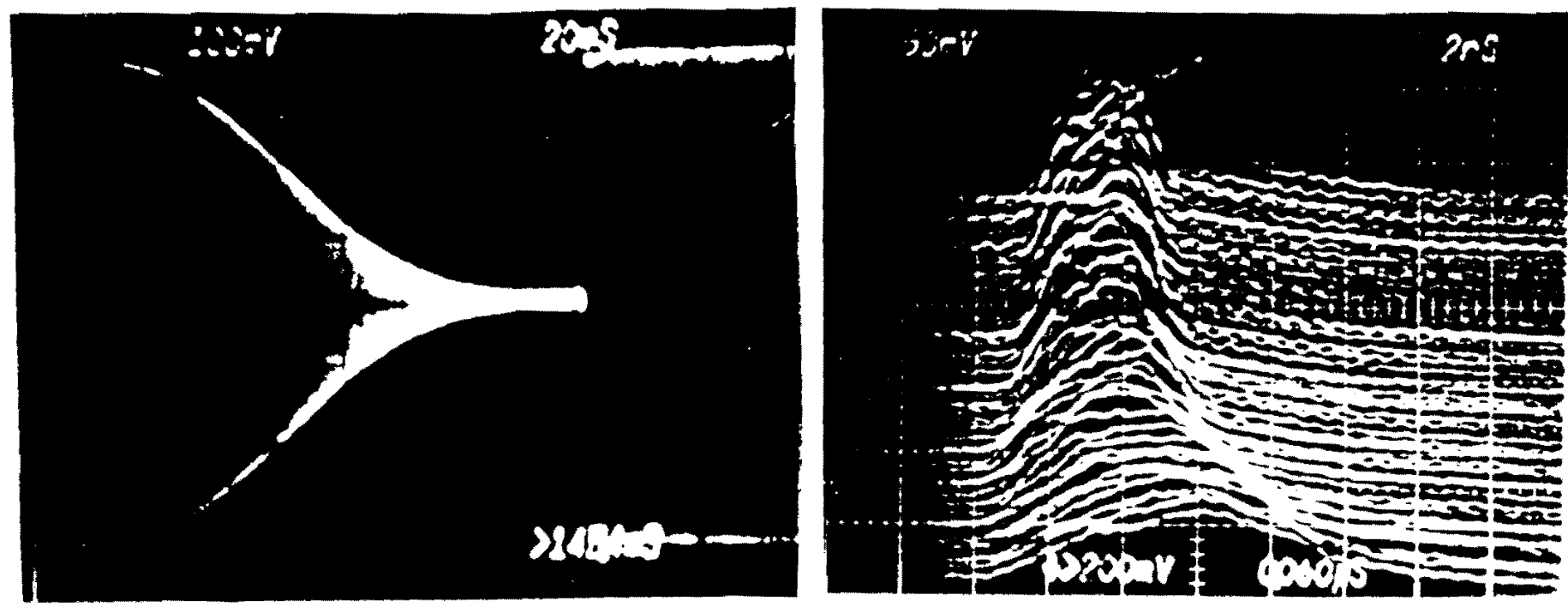

(a)

(b)

Figure 8

RF voltage reduction (a) and bunch shape (b) just prior to raising $r f$ voltage for $\overline{\mathrm{p}}$ production.

Bucket area reduction on flattop for coalescing small ensembles of protons or $\bar{p}$ 's will require reduction of the rf voltage to a value

considerably lower than that cequired for $\bar{p}$ production. Tn the case of eleven proton bunches, each with $3=0.1$ eV-sec, a final rf voltage of $1 \mathrm{kV}$, providing a bucket area of 0.13 eV-sec will be required. If each bunch

contains $9 \times 10^{9}$ protons the maximum bean induced voltage during ensemble passage will be about $2.5 \mathrm{kV}$. It appears very difficult to develop stable voltages at this level using counter-phased high level rf alone.

An additional relatively small, low impedance, easily controllable re systen capable of developing $8-10 \mathrm{kV}$ could be operated in conjunction with the counter-phased $\mathrm{rf}$ to provide very low voltage. Such a system could be buit. using surplus $\mathrm{M}_{4} \mathrm{C}_{21 \mathrm{~A}}$ ferrite cores. Each core, operated at an $\mathrm{r}$ flux of $30 \mathrm{mi}^{\mathrm{i}}$ would develop about $1 \mathrm{kV}$ at $53 \mathrm{MHz}$ and dissipate about 75 watts. The $Q$ of such a system would be about 100. A "cavity" would consist of a ceramic gap and four cores (or eight cores in a back-to-bact: double ended configuration with one gap). The system could be driven with several 300 watt broadband rf amplifiers or a single $1 \mathrm{kw}$ narrow band amplifier, depending on details of how the structure is to be operated. Tf broadband amplifeiers are used the system might also provide some

longltuitinal damping at other times. 
Since the rf fan-back voltage may not provide a rellable indication of the gap voltage due to limiations in calibration, it may be reasonable to try to develop techniques for using the beam itself as an indication of the rf voltage level. For instance, suppose beam bunch signals are passed through a broadband $(1 \mathrm{GHz})$ two-way splitter and each of the outputs is passed through a narrow band filter, one centered on the fundamental frequency $(53 \mathrm{MHz})$ and the other centered on the second harmonic frequency $(106 \mathrm{MHz})$. The two outputs can then be diode detected and the second harmonic signal divided by the fundamental signal in an analog divider. If the bunches were very narrow (delta functions) the ratio of these two harmonics would be one so this technique is automatically normalizing, independent of beam intensity. ( $I$ am lgnoring the effects of the filters, which will introduce some additional Fourler transform fooling around). Let the bunches be represented by expression

$$
\begin{aligned}
i(t) & =i_{p} \cos ^{2}\left(\frac{\pi t}{W}\right) & & -\frac{\pi}{2}<\frac{\pi t}{W}<\frac{\pi}{2} \\
& =0 & & \text { elsewhere }
\end{aligned}
$$

(This is a Gaussian-like distribution which also represents a "parabolic Ine distribution" with small talls which terminate with zero slope. Very useful). W is the full bunch length, assumed here to be less than $T$, the rf perlod or buoket length. If we express the bunch length $W$ as a fraction $k$ of the bucket length $\left(W=k T_{r f}, k<1\right)$ then harmonic component ratio $\mathrm{k}$ becomes

$$
R=\left(\frac{1-k^{2}}{1-4 k^{2}}\right) \cos k \pi
$$

Th1s function progresses smoothly from one to zero as the bunch length goes from a delta function to full bucket length after which (larger values of k) it oscillates about zero with amplitude approaching 0.25 . For debunching to full buckets (required for proton coalescing) the voltage ratio $\mathrm{R}$ could be compared to a debunching program or model, and the difference used to modulate the amplitude of the small vernier cavities used in conjunction with the counter-phased high level rf. Using some technique like this it appears feasible to control the voltage automatically using signals derived from the beam but some stability problens may arise if the counter-phased high level voltage component were to pass through zero and come up to some small level of opposite phase. Some ingenious technlque should be invented to prevent this from happening.

Further reduction in net $r f$ voltage, i.e. that required for debunching of $\bar{p}$ bunchees into matched $h=53$ buckets prior to bunch rotation for coalescing can be controlled using the vernier cavity system with 
amplitude controlled by signals derived from the ratio of the $h=53$ Fourier component and the $\mathrm{h}=1113$ component in a manner simflar to that suggested above. Detailed expressions for these components will be presented elsewhere.

The $h=53 \mathrm{rf}$ voltage required to generate the small bucket into which $\bar{p}$ 's are debunched prior to coalescing is so low (approx. 100 volts) that it may be difficult to generate using the large coalescing cavities. Again it may be necessary to provide a small ferrite loaded cavity capable of generating nominally 100 volts at $2.5 \mathrm{MHz}$ and driven by a dedicated relatively low power amplifier.

5. Azimuthal Position Adjustment of Main Ring Bunches

After bunches are coalesced on Main Ring flattop at $150 \mathrm{GeV}$ (possibly before for some reason) they will usually have to be moved azlmuthally with respect to bunches which have been previously injected into the Tevatron. In order to do this the two systems should first be phase-locked together and the time delay (number of rf perlods) between passage of the Main Ring bunch and the appropriate Tevatron bunch should be measured using signals from directional couplers in each machine. From this information the number of degrees of phase advance required in the Main Ring can be calculated. The required phase advance can be introduced elther by a smooth frequency excursion of the master oscillator or by directly exciting the phase shifters used for counter-phasing of the rf. In any case the correction should be done slowly and smoothly. The frequency excursion assoclated with the required phase advance should be limt ted to that which keeps the beam within some useful momentum aperture. The frequency range limitation will place a constraint on the time required to do the phase adjustment but it does not appear to be a significant limitation.

Because the identity of the beam phase may become obscured during these processes, it may be reasonable to perform these functions with the beam phase and position feedback loops disabled. This could be accomplished by switching the rf source to a fixed frequency oscillator at a point beyond the operational phase shifter. The beam bunches and the flxed frequency osclilator must be phase locked together prior to this switch, and in the interest of minimizing the resulting beam disturbance it would seem reasonable to phase lock the osclllator to the beam phase rather than the other way around prior to switching. If the centered beam frequency differs from the target osclllator frequency (because the beam energy and magnet fleld are not quite correct) the frequency could be allowed to move slowly (w1th respect to synchrotron period) to the target frequency after the switch but before the counter-phasing operation.

The beam loading compensation voltage must be introduced into the fan-out system at two points following the counter-phasing devices or the beam loading compensation will be counter-phased out just when it is most needed. 
$\overline{\mathrm{p}}$ Note 382

J.E. GriffinJ. MacLachlan

May 1984

\section{Some Suggested Techniques for Achieving Required Main Ring Low Level RF Performance for the Tevatron I Program}

These techniques are derived from experience with the existing low level rf system and from the results of accelerator experiments directed at the Tev I requirements which were performed using a speclally modifled low level rf system. The techniques suggested here are not necessarily unique and other techniques may be acceptable.

It is important to realize that certain performance requirements on the Booster and Main Ring are substantially different in the Tev I program from those which have been acceptable in the fixed target program. For example, the longitudinal emittance of the bunches has been routinely and intentionally blown up by the "bunch spreader" during acceleration to improve the smoothness of the spill. In the Tev I case, the "bunch narrowing" procedure preceding $\bar{p}$ production and in bunch coalescing is facilitated by maintaining the smallest possible longitudinal emittance (i.e. the largest possible longitudinal phase space density). Also, during fixed target physics the Main Ring is essentially full (i.e. approx. 1090 of 1113 buckets occupled) so transient beam loading of the $r f$ system is negligible except during injection, whereas for $\bar{p}$ production a single high intensity Booster batch (approx. 83 bunches) is to be accelerated causing substantial transient beam induced phase shift of the rf voltage during each passage. The low level phase and position error signals are normally derived from an essentially full ring during fixed target operation whereas in the Tev I program the systems must perform adequately with as few as ten adjacent buckets occupied.

Because of the differences in emphasis between fixed target operation and Tevatron I operation is seems sensible to use separate low level rf systems for these two modes of operation. If a single low level system were to be used for both modes of operation some compromises may be necessary which might cause performance in either mode to be less than adequate. Because of the severe demands made on accelerator performance in the Tev I program it would seem reasonable to develop a unique system which can be optimized to the requirements.

1. Longitudinal Dilution at Injection

In normal operation the Main Ring rf frequency is held fixed during thirteen Booster acceleration cycles and the Booster rf is phase-locked to the fixed frequency during the last $3-5 \mathrm{msec}$ of each cycle. These Booster phase-locks cause a variable amount of dilution, depending upon somewhat random initial conditions, on each cycle. This procedure is necessary because of the succession of thirteen Booster batches required to fill the 
Main Ring. In the Tev I program only one (or less than one) Booster batch is accelerated on each cycle so the procedure can be reversed; the Main Ring frequency and phase can be locked to the Booster near the end of the Booster cycle. This will eliminate the rapid and somewhat variable bucket motion just prior to Booster extraction. It should be possible to adjust the phase and amplitude of the Main Ring buckets so that the injected bunches are accurately matched to the buckets. At the time of Booster extraction the Main Ring-Booster phase-lock should be terminated and the Main Ring frequency and phase held fixed until well established beam induced feedback signals can be applied.

The same procedure should be applied when antiprotons are injected from the Accumulator ring except that the Main Ring should then be locked to the Accumulator rf.

It seems reasonable to use the same phase detector to accomplish these two functions with an $\mathrm{rf}$ switch to select the $\mathrm{rf}$ source to which the system is locked. An additional switch position could select an rf signal derived from beam in the Main Ring at an appropriate time. With an appropriate transfer function the transient disturbance delivered to the VCO when $\mathrm{rf}$ sources are switched could be minimized.

An even simpler scheme, involving no switches is shown in Figure 1. An amplified and gain-controlled signal derlved from the Booster beam is delivered to a combiner, the output of which is delivered to the phase detector and compared to the delayed MRRF VCO output (such a signal exists in the Booster low level of system). In this way the Main Ring of is locked to the Booster beam just prior to injection. A signal from the Main Ring beam plckup, suitably amplified and leveled, is delivered to another input of the combiner. When beam is extracted from the Booster the Booster signal disappears and a few microseconds later a slgnal appears from the Main Ring pickup. With phases properly adjusted a smooth transfer of phase lock with minimum transient disturbance should occur automatically.

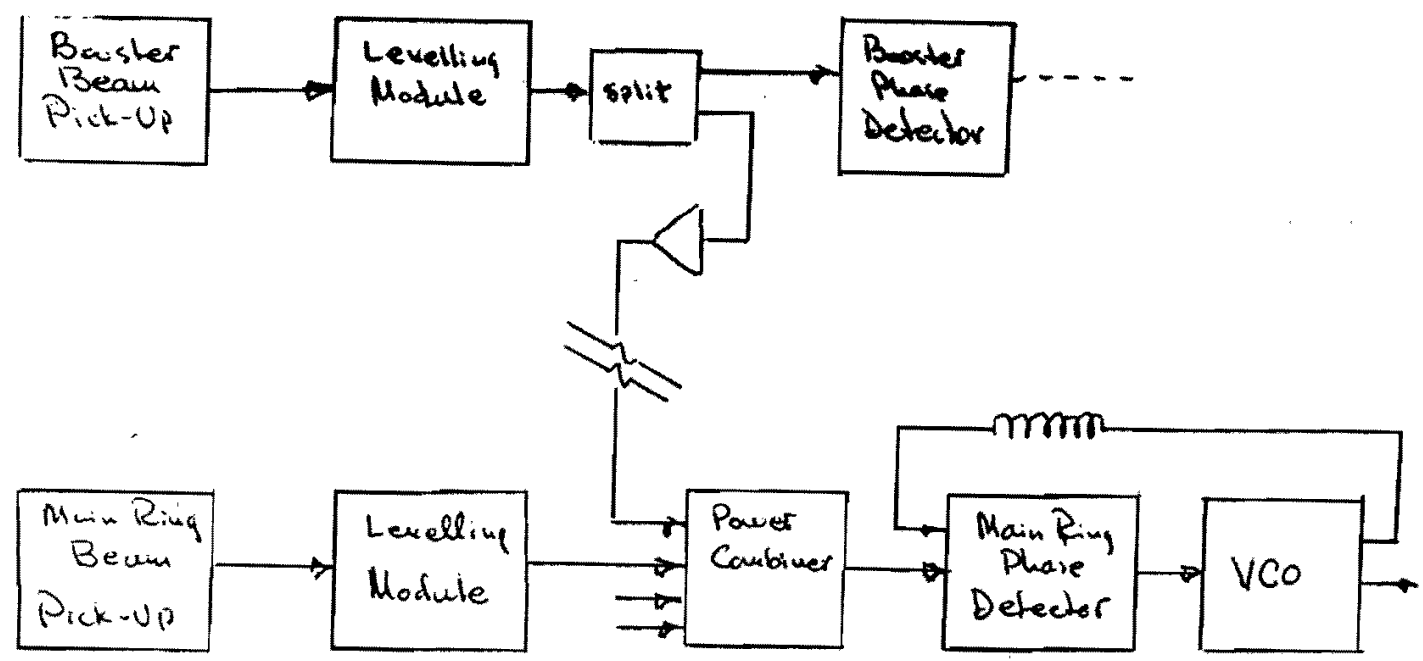

Figure 1 
Signals from other sources, such as the Antiproton Accumulator Ring can be delivered to the combiner in a similar way. There may be additional technical complexity relating to the number of bunches involved or unwanted signals so some additional gating may be necessary.

Alternatively, several phase detectors, each dedicated to a particular function, could be employed. It would seem that the likelihood of transient disturbance of the VCO at switching times would be worse in this configuration but it may be possible to avold this problem in some way. This choice would appear to require significantly more bin space than using rf switching. If more than one or two phase detectors are to be switched into the VCO the problem of the offset voltages between units may become difficult to manage.

2. Small Ensemble Acceleration

In the acceleration of an ensemble of a few bunches (approx. 10) or a single Booster batch it is important that the error signal from the phase detector delivered to the VCO remain at an acceptably stable and constant value during those periods (approx. 20 microseconds each turn) when beam signals are absent. A simple approach to the problem is to use a Track and Hold element between the phase detector and the VCO.

Unfortunately this technique is particularly difficult when the $T \& H$ system is used to deliver an error signal in a feedback loop, as is required here, because the error signal is nominally zero and the $T \& H$ offset voltage or "pedestal" may be comparable in magnitude to a significant error voltage. One way to manage this problem is to amplify the error signal as much as is practical ahead of the $T \& H$ and to follow the T\&H with equivalent attenuation. Since the pedestal is constant its effect is reduced by the attenuation. Another approach is to digitize the error signal and deliver updating digital information to a digitally controlled variable frequency oscillator. "Hold" is accomplished by terminating the updating process.

Suppose, as an example, that the "track to hold" offset voltage is 25 $\mathrm{mV}$. If the $V C O$ has a frequency to voltage sensitivity of $50 \mathrm{kHz}$ per volt the offset will result in a frequency shift of $1250 \mathrm{~Hz}$ or an angular frequency of $2500 \pi$ radians per second. In the $19.3 \mathrm{microsecond} \mathrm{period}$ between passage of single batches this frequency shift will result in a phase shift (compared to the "correct" frequency with no frequency shift) of about $0.05 \pi$ radians or 9 degrees. A phase shift of this magnitude in the bucket location with respect to the returning beam would be a matter of concern. Fortunately, the rf cavities act as a flywheel and they cannot respond fully to this drive phase change in one rotation period. Nevertheless such a phase shift is not negligible and some consideration should be given to reducing or eliminating it.

The existing radial position modules have a gating feature which is ideally suited to operation of a small ensemble $T \& H$ unit. This signal is 
called the "mode" signal. The signal is shown in Figure 2 along with the rf output of the leveling module. The signal starts in the high or "track" condition, goes low at the end of ensemble passage, then goes high on each successive passage. Because of the relative location of the pickups involved the relative times of the signals are ideal. Unfortunately this time relationship will not be preserved for antiprotons golng the other direction so some other source of gating information may be required.
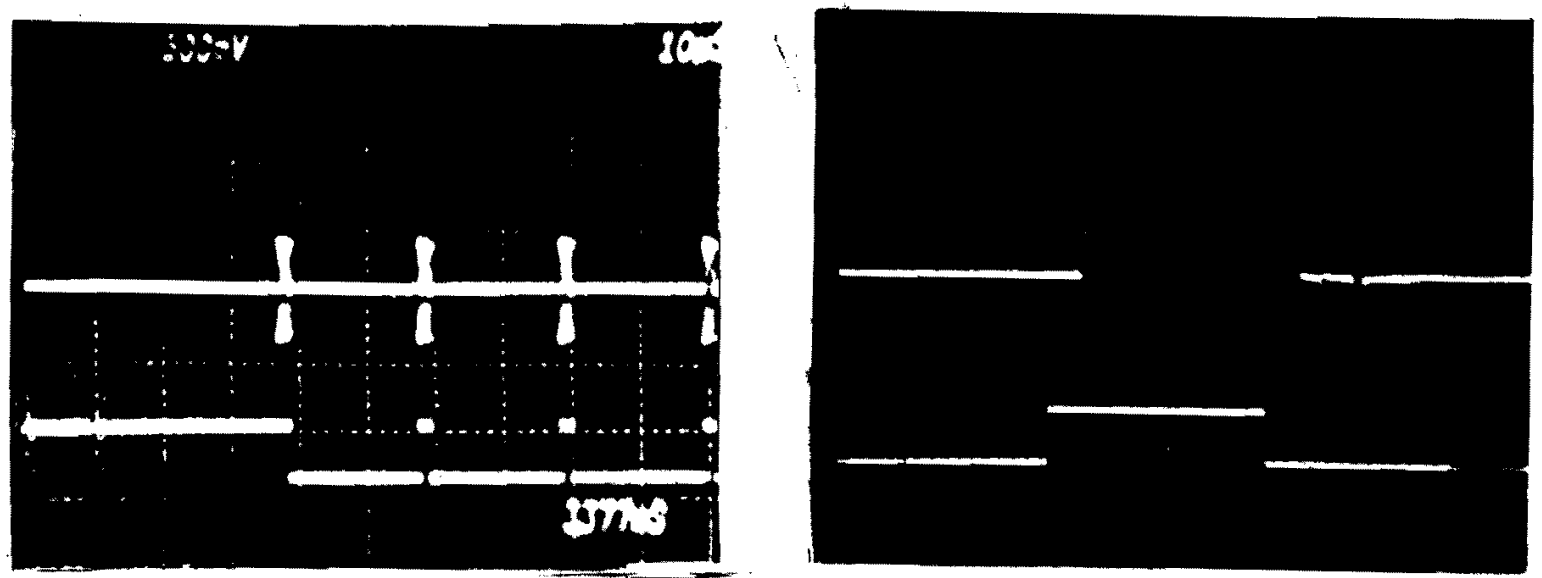

Figure ?

"Mode" signal from radial position system, with beam signal from leveling module.

\section{Translent Beam Loading}

A single Booster batch with 84 bunches each containing $g 2.5 \times 10^{10}$ protons will induce a voltage in the Main Ring rf system varying from zero to about $100 \mathrm{kV}$ during a 1.6 microsecond passage. This transient voltage will decay to about half this value during the 19.3 microseconds while the batch is elsewhere and recur at each batch passage of the rf cavities. In a stationary bucket situation with nominally $10^{6}$ volts developing buckets, this transient voltage will cause a phase shift of about 6 degrees. This means that some bunches in the batch will be mismatched to the bucket center and the ensuing coherent dipole motion will cause a longitudinal density dilution. Figure 3 shows two traces of the phase shift resulting from passage of a single batch (approx. 84 bunches) of approx. the intensity mentioned above through the Main Ring rf system at $8 \mathrm{Gev}$ while the system was developing about $1 \mathrm{MV}$. The two traces were separated by adding a length of line corresponding to 7.2 degrees of phase shift. The transient beam loading phase shift is about 5 degrees. 


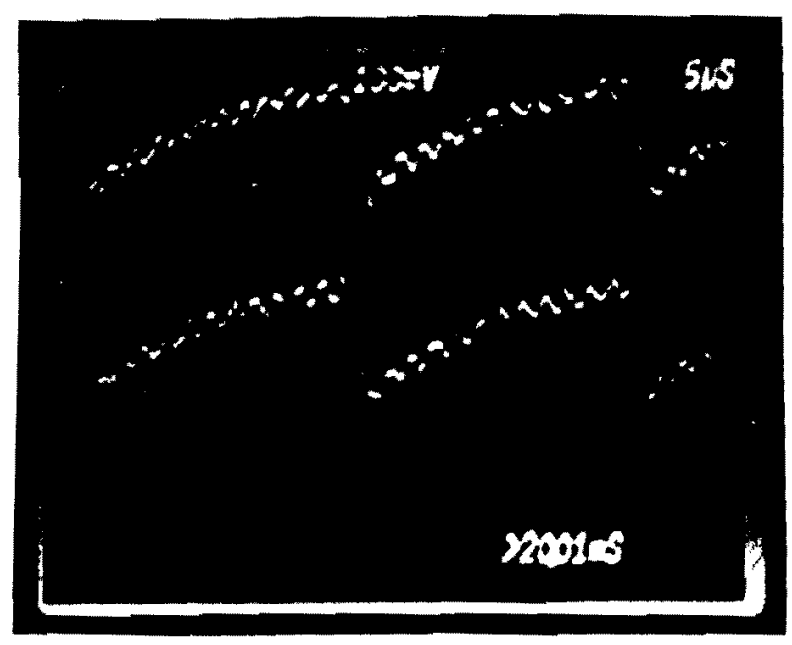

Eigure 3

Phase Shift in the Main Ring rf voltage resulting from passage of a single Booster batch of beam through the Main Ring rf system at $8 \mathrm{GeV}$.

During acceleration the transient voltage will cause a variation in bucket area over the batch in addition to the phase shift.

In principle the transient beam loading could be eliminated by deliverying to the rf cavities a burst of current equal and opposite in time and amplitude to the $53 \mathrm{MHz}$ Fourier component of the passing beam bunches. There is no simple way to do this directly although one could think of pickups located part way around the ring and direct cables to Fo. A simple solution would be to sample the beam passage at Fo, remember the information for one turn (about 20 microseconds) and introduce compensating current derived from the information into the rf system always one turn late. Unfortunately there is a large quantity of information contained in 84 bunches and there are technical difficulties associated with remembering accurately the information for 20 microseconds and returning it to the system. Since the cavities respond only to the $53 \mathrm{MHz}$ component of the beam current (we suppose), it is necessary to remember only the amplitude, frequency, and phase of this component. The held VCO output frequency does part of this job, remembering the frequency and phase, subject to the $T \& H$ pedestal and drift errors mentioned earlier. To a large extent the transient beam loading can be compensated for in the low level rf system by feeding a burst of the VCO output signal around the operational phase shifters (including counter-phase modules) and adding 1 t to the fan-out voltage. The signal must be gated to the rf cavities only when the beam is passing and its amplitude must be adjusted to the beam intensity. An appropriately timed gating signal can be developed using the $47 \mathrm{kHz}$ rotation frequency clock and an adjustable delay. The amplitude of the signal fed forward should be adjusted automatically to match the beam 
intensity on each acceleration cycle. The vCO output rf is alway phase-locked to the beam and fixed phase shifts are introduced such that when the operational phase shifter is not driven (i.e. Input voltage is zero, corresponding to zero relative phase shift) the rf cavities develop a stationary bucket. At injection, below transition, the beam current leads the $\mathrm{rf}$ gap voltage in phase by $\pi / 2$ radians. If the fed forward voltage is to excite the rf cavities at a phase opposite to the beam excitation then the phase must lag the VCO voltage delivered to the operational phase shifter by an additional $\pi / 2$ radians.

In Figure 4 the fan-out fan-back phase shift with and without compensation is shown for a single Booster batch. Figure 5a shows the relative gating time in relation to the beam signal from the leveling module at the low level rf bin location. Figure $5 \mathrm{~b}$ shows the fan-out rf signal during this compensation. During this experiment application of the compensation caused a repeatable 13 percent increase in beam intensity at $100 \mathrm{GeV}$ for normalized injection conditions.

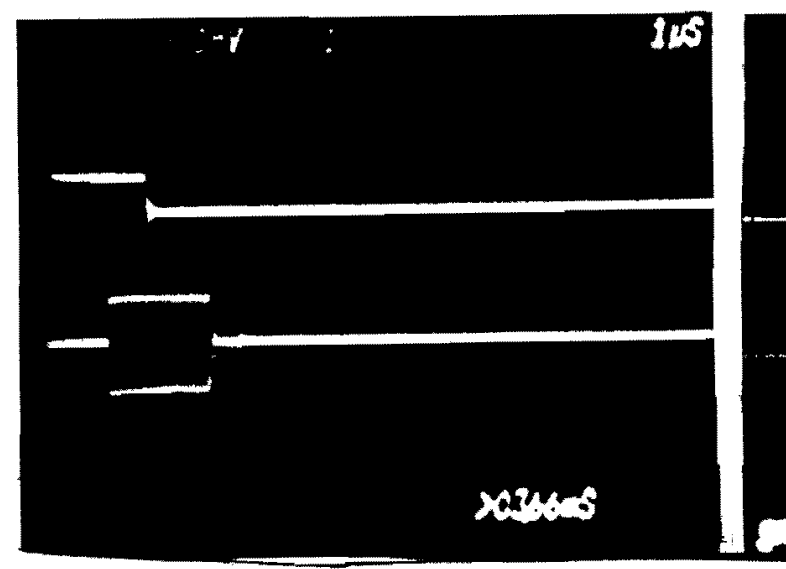

(a)

\section{FIGURE 4}

Improvement in fan-out fan-back beam induced phase shift with feed-forward compensation.
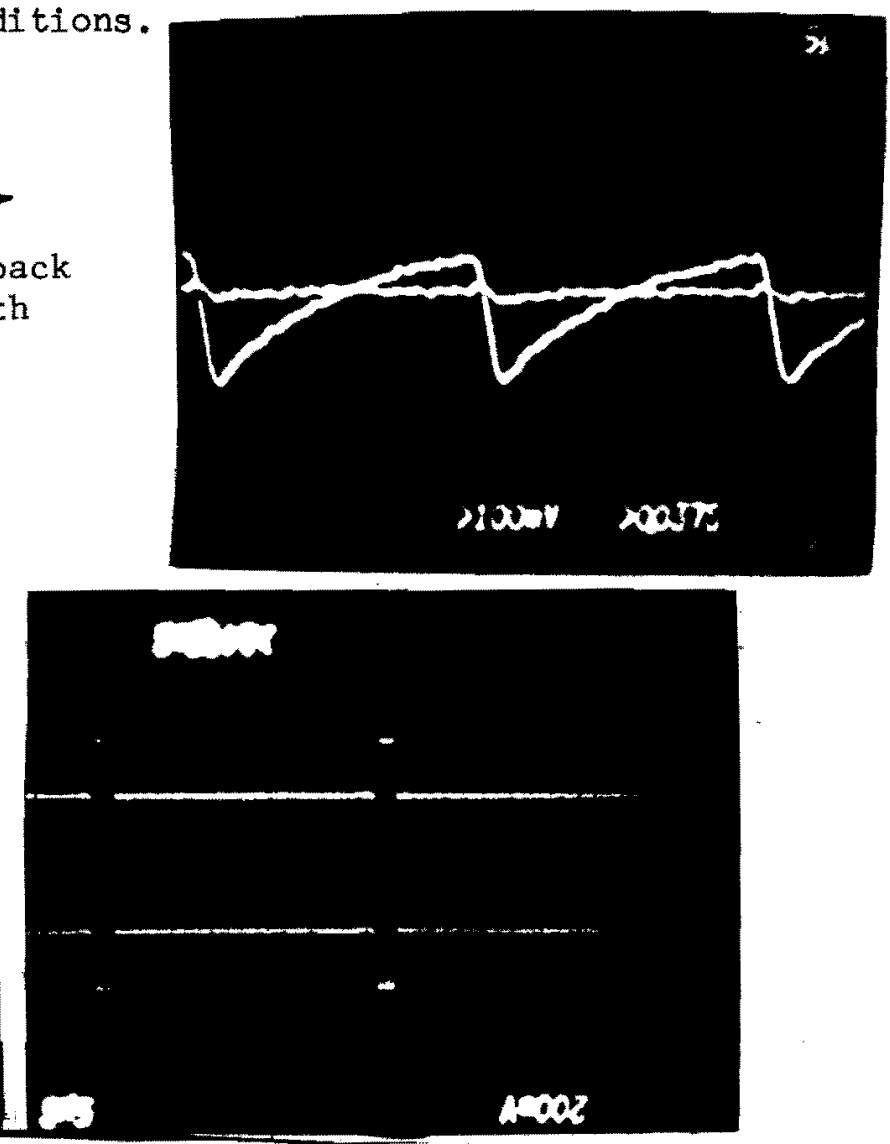

(b)

Figure 5

(a) Optimum gating timing for compensation with respect to beam signal from leveling module. (b) Fan-out $r f$.

The increase in beam intensity presumably resulted from improved passage through transition of bunches with reduced longitudinal dilution. 
The procedure outlined above for transient beam loading compensation will probably work adequately for single Booster batch acceleration. Bunch lengths during these operations remain reasonably constant and the $53 \mathrm{MHz}$ component of the distributions remains reasonably constant. Several problems may arise during flattop operations. First the rf is to be derived from a flattop oscillator rather than from the VCO and some problems may arise in feeding the VCO signal forward and adding it to the fan-out rf. In principle this should work as before because the vco should be phase-locked to the beam regardless of the actual source of $\mathrm{rf}$. In practice this phase-lock may not be adequate for some unexpected reason.

The second problem arises during $\mathrm{rf}$ voltage reduction for bunch broadening. For a fixed intensity the $53 \mathrm{MHz}$ Fourier component of beam exclting the rf system will decrease as the bunches are broadened. While this decrease may only be a factor of about 0.75 it may still cause a compensation error just when the required resultant voltage is decreasing to an amplitude which is, in fact, smaller than the beam induced voltage. A Booster batch with $2 \times 10^{10}$ protons per bunch and $S=0.3 \mathrm{eV}-\mathrm{sec}$ per bunch (design study values) can induce a maximum voltage of about $45 \mathrm{kV}$ in the system while the required voltage is about $34 \mathrm{kV}$. If the anode program and cascode current on each rf cavity is reduced to too low a value during counter-phasing, there may not be enough rf amplifier current avaliable to compensate for beam loading even if the fed-forward signal is at the "correct" value.

Two examples of counter-phased rf voltage with improperly adjusted compensation and one example of approximately correct compensation are shown in Figure 6. 

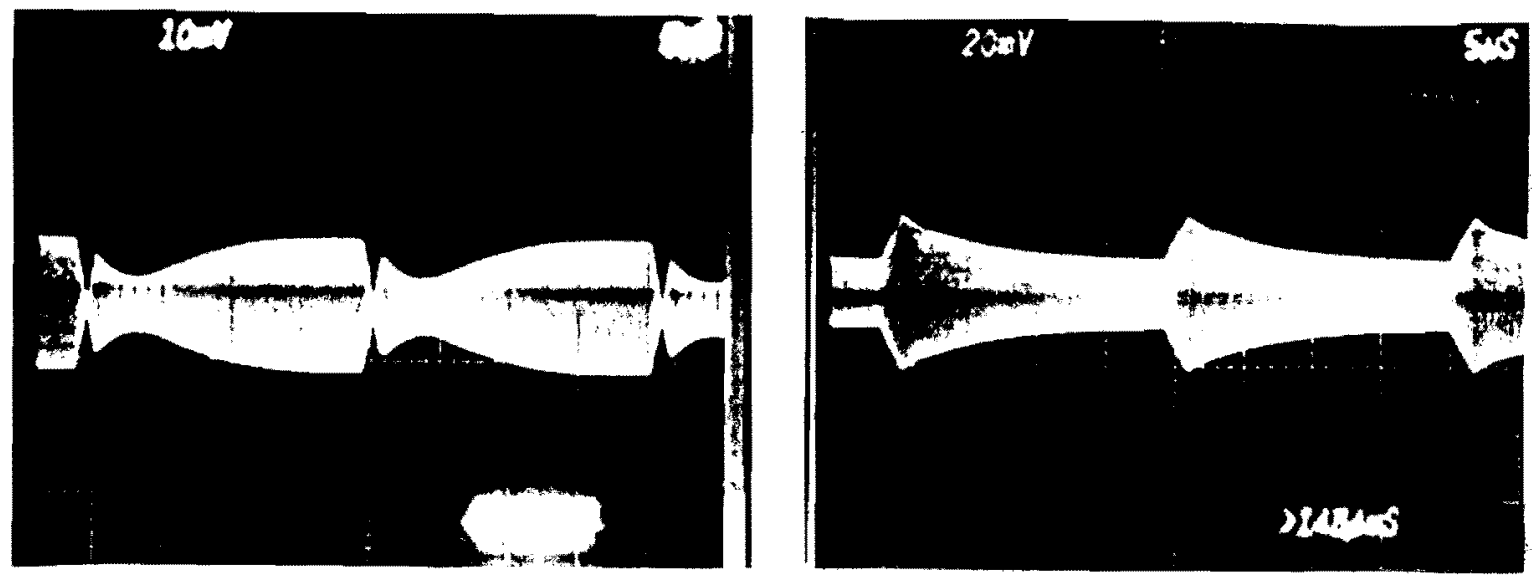

(a)

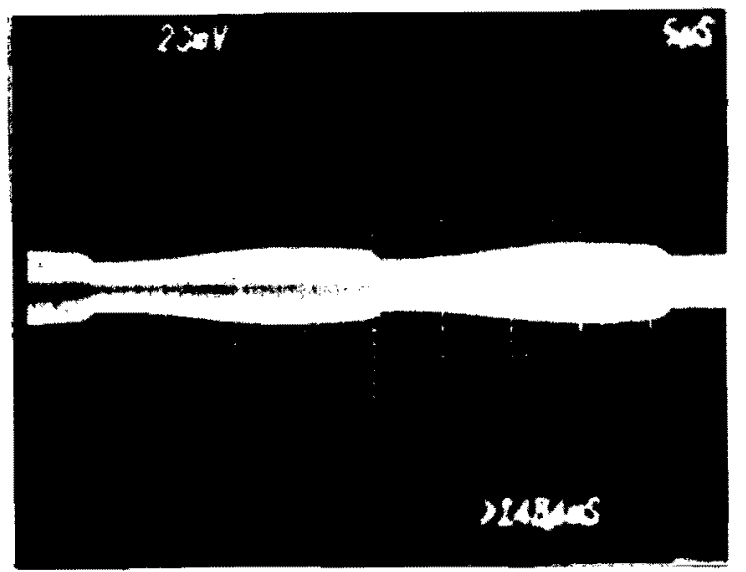

(b)

\section{Figure 6}

(a) Improperly adjusted transient beam loading rf voltage during counter-phasing. (b) Approximately correct adjustment of transient beam loading compensation

during $\mathrm{rf}$ counter-phasing.

Transient beam loading from small ensembles during adiabatic debunching prior to and during coalescing at $h=53$, may present a more difficult problem. The "debunched" small ensembles may, in some configurations, develop voltages in the rf system which are comparable to or larger than those required to debunch the beam. These voltages may be developed even when the MR cavities are off during bunch rotation at $h=53$. This situation begins to look like an instability rather than $a$ standard beam loading problem, and a separate document treating the problem is being prepared.

4. RF Voltage Reduction on Flattop

During $\overrightarrow{\mathrm{p}}$ production and during the bunch coalescing process necessary to produce intense $p$ and $\bar{p}$ bunches the rf voltage must be reduced to very 
small values during the $120 \mathrm{GeV}$ and $150 \mathrm{GeV}$ flattops. It seems reasonable to accomplish this reduction by counter-phasing approximately equal numbers of $\mathrm{rf}$ cavities while at the same time reducing the voltage developed by each cavity to the smallest possible value. At least one and probably two feedback loops (on phase and amplitude) will be required to control the amplitude and maintain the proper phase of the reduced bucket.

In the $\overline{\mathrm{p}}$ production mode the net rf voltage creating stationary buckets must be reduced to the order of $10 \mathrm{kV}$ before it is raised to its maximum value (about $4 \mathrm{MV}$ ). About $100 \mathrm{msec}$ will be required for this reduction and the voltage proflle is dictated by the requirement of adiabaticity and minimum dilution. At the end of the voltage reduction the bunch should extend over a range $\pm \pi / 2$ radians of $\mathrm{rf}$ phase. In order to reduce the bucket area smoothly with a minimum of dilution, the incremental change in bucket area, dS, should be adjusted to be a constant fraction of the phase oscillation period $\tau$. This condition can be described by an adiabaticity parameter $\alpha$,

$$
\alpha=\text { const. }=\frac{\tau}{\mathrm{S}} \frac{\mathrm{dS}}{\mathrm{dt}}
$$

The ratio $\alpha$ should be small for small dilution but empirically it is found that it can be as large as 2 without serious dilution. The resulting voltage program can be expressed

$$
V(t)=\frac{1}{\left(1-\frac{\alpha t}{\tau}\right)^{2}}
$$

where, for a process taking $N$ turns

$$
\alpha=\left[1-\left(V_{0} / V_{N}\right)^{1 / 2}\right] \frac{T_{0}}{N \tau}
$$

\footnotetext{
where: $V_{0}=r f$ voltage at start

$\mathrm{V}_{N}^{\circ}=\mathrm{rf}$ voltage after $\mathrm{N}$ turns

$T_{0}^{N}=$ rotation period of Main Ring

$\tau_{0}^{\circ}=$ synchrotron phase oscillation period at start.
}

In the design study case, $2 \times 10^{10}$ protons per bunch, each with $S=0.3$ $\mathrm{eV}-\mathrm{sec}$ the final value of the $\mathrm{rf}$ voltage required to spread the bunches over $\pm \pi / 2$ radians is about $35 \mathrm{kV}$. The phase oscillation period at $1 \mathrm{MV}$ and 
$120 \mathrm{GeV}$ is $0.01 \mathrm{sec}$ so an adiabaticity factor of $\alpha$ of -1 results in a debunching period of about two thousand turns or about $42 \mathrm{msec}$. The time dependence of the $\mathrm{rf}$ amplitude is

$$
V(t)=\frac{1}{(1+100 t)^{2}} \mathrm{MV}
$$

The expression for $\alpha$ shows that it is only weakly dependent on $v_{0}$ if $V_{0} \gg V_{N}$. Therefore it matters little whether the starting voltage is $1 \% \mathrm{MV}$ or as high as $4 \mathrm{MV}$. However, if an attempt has been made to minimize dilution during the final phase of acceleration by keeping the bunch length nearly constant during the field parabola from acceleration to flattop, the rf voltage will have been reduced to about $1 \mathrm{MV}$, as used in the above example.

A plot of a voltage reduction curve with adiabaticity factor -0.5 is shown in Figure 7. The curve starts at $1 \mathrm{MV}$ and it is followed by a fast excursion to $4 \mathrm{MV}$. 


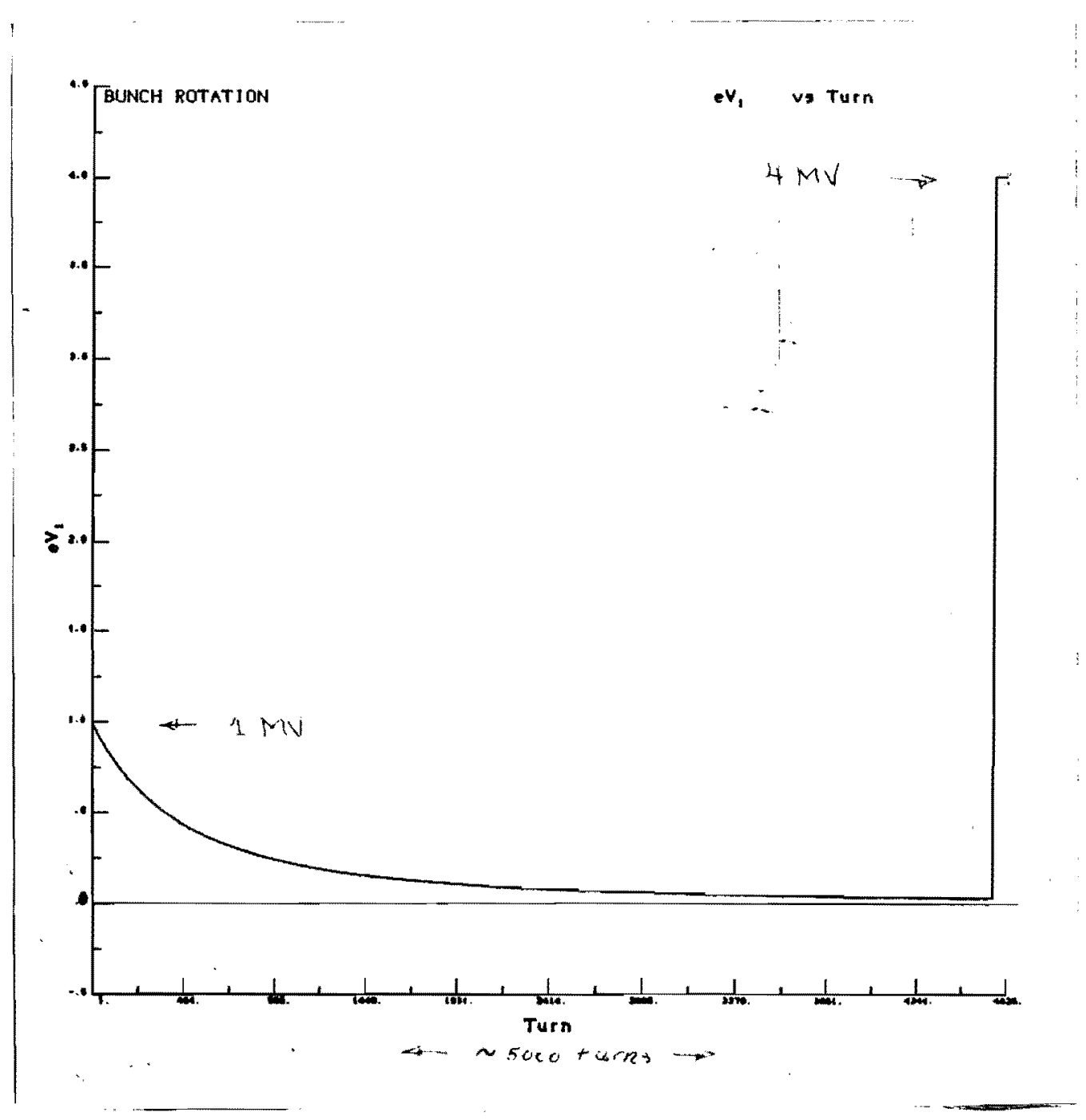

Figure 7

Adiabatic $r f$ voltage reduction curve for $\bar{p}$ production.

At the end of the voltage reduction phase the voltage must be raised as quickly as possible to the maximum possible value and held at that level for one quarter of a synchrotron perlod. At $4 \mathrm{MV}$ and $120 \mathrm{GeV}$ the synchrotron period is about $5 \mathrm{msec}$ so the beam will be extracted about 1.3 msec after the voltage is raised.

In feasibility experiments bunch lengths of 1 nsec full width were obtained using the above described procedure and a maximum rf voltage of $3.5 \mathrm{MV}$. The results Indicated that the longitudinal enittance of the bunches in the experiment were about $0.3 \mathrm{eV}-\mathrm{sec}$ and a counter-phase $\mathrm{rf}$ voltage of about $26 \mathrm{kV}$ was sufficient to spread the bunches over half the bucket length. In Figure $8 \mathrm{a}$ and $8 \mathrm{~b}$ the $\mathrm{rf}$ voltage reduction profile and the resulting bunch shape are shown prior to sudden raising of the voltage. 

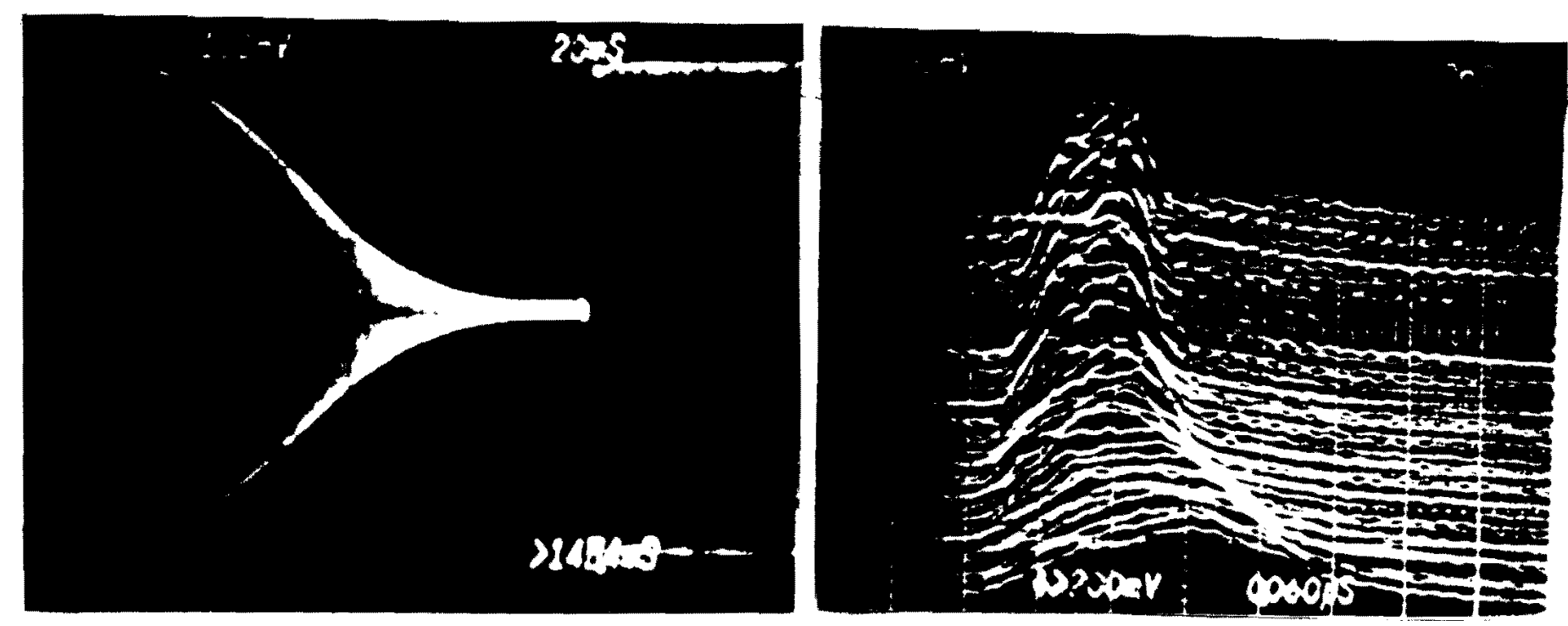

(a)

(b)

Figure 8

RF voltage reduction (a) and bunch shape (b) just prior to raising $\mathrm{rf}$ voltage for $\bar{p}$ production.

Bucket area reduction on flattop for coalescing small ensembles of protons or $\bar{p}^{\prime} s$ will require reduction of the $r f$ voltage to a value considerably lower than that required for $\bar{p}$ production. In the case of eleven proton bunches, each with $S=0.1 \mathrm{eV}-\mathrm{sec}$, a final rf voltage of $1 \mathrm{kV}$, providing a buckgt area of $0.13 \mathrm{eV}-\mathrm{sec}$ will be required. If each bunch contains $9 \times 10^{9}$ protons the maximum beam induced voltage during ensemble passage will be about $2.5 \mathrm{kV}$. It appears very difficult to develop stable voltages at this level using counter-phased high level $r f$ alone.

An additional relatively small, low impedance, easily controllable rf system capable of developing $8-10 \mathrm{kV}$ could be operated in conjunction with the counter-phased $\mathrm{rf}$ to provide very low voltage. Such a system could be built using surplus $\mathrm{M}_{4} \mathrm{C}_{21 \mathrm{~A}}$ ferrite cores. Each core, operated at an $\mathrm{rf}$ flux of $30 \mathrm{mT}$ would develop about $1 \mathrm{kV}$ at $53 \mathrm{MHz}$ and dissipate about 75 watts. The $Q$ of such a system would be about 100 . A "cavity" would consist of a ceramic gap and four cores (or elght cores in a back-to-back double ended configuration with one gap). The system could be driven with several 300 watt broadband $\mathrm{rf}$ amplifiers or a single $1 \mathrm{kw}$ narrow band amplifier, depending on details of how the structure is to be operated. If broadband amplifelers are used the system might also provide some longitudinal damping at other times. 
Since the rf fan-back voltage may not provide a reliable indication of the gap voltage due to limiations in calibration, it may be reasonable to try to develop techniques for using the beam 1 tself as an indication of the rf voltage level. For instance, suppose beam bunch signals are passed through a broadband ( $1 \mathrm{GHz}$ ) two-way splitter and each of the outputs is passed through a narrow band filter, one centered on the fundamental frequency ( $53 \mathrm{MHz}$ ) and the other centered on the second harmonic frequency $(106 \mathrm{MHz})$. The two outputs can then be diode detected and the second harmontc signal divided by the fundamental signal in an analog divider. If the bunches were very narrow (delta functions) the ratio of these two harmonics would be one so this technique is automatically normalizing, independent of beam intensity. ( $I$ am ignoring the effects of the filters, which will introduce some additional Fourier transform fooling around). Let the bunches be represented by expression

$$
\begin{aligned}
i(t) & =i_{p} \cos ^{2}\left(\frac{\pi t}{W}\right) & & -\frac{\pi}{2}<\frac{\pi t}{W}<\frac{\pi}{2} \\
& =0 & & \text { elsewhere }
\end{aligned}
$$

(This is a Gaussian-like distribution which also represents a "parabolic line distribution" with small tails which terminate with zero slope. Very useful). W is the full bunch length, assumed here to be less than $T$, the rf period or bugket length. If we express the bunch length $W$ as a fraction $\mathrm{k}$ of the bucket length $\left(\mathrm{W}=\mathrm{kT} \mathrm{rf}_{\mathrm{f}}, \mathrm{k}<1\right)$ then harmonic component ratio $\mathrm{R}$ becomes

$$
R=\left(\frac{1-k^{2}}{1-4 k^{2}}\right) \cos k \pi
$$

This function progresses smoothly from one to zero as the bunch length goes from a delta function to full bucket length after which (larger values of k) it oscillates about zero with amplitude approaching 0.25 . For debunching to full buckets (required for proton coalescing) the voltage ratio $\mathrm{R}$ could be compared to a debunching program or model, and the difference used to modulate the amplitude of the small vernier cavities used in conjunction with the counter-phased high level rf. Using some technique like this it appears feasible to control the voltage automatically using signals derived from the beam but some stablifty problems may arise if the counter-phased high level voltage component were to pass through zero and come up to some small level of opposite phase. Some ingenious technique should be invented to prevent this from happening.

Further reduction in net of voltage, $i . e$. that required for debunching of $\bar{p}$ bunchees into matched $h=53$ buckets prior to bunch rotation for coalescing can be controlled using the vernier cavity system with 
amplitude controlled by signals derived from the ratio of the $h=53$ Fourier component and the $h=1113$ component in a manner similar to that suggested above. Detalled expressions for these components wll be presented elsewhere.

The $\mathrm{h}=53 \mathrm{rf}$ voltage required to generate the small bucket into which $\bar{p}^{\prime}$ s are debunched prior to coalescing is so low (approx. 100 volts) that it may be difficult to generate using the large coalescing cavities. Again it may be necessary to provide a small ferrite loaded cavity capable of generating nominally 100 volts at $2.5 \mathrm{MHz}$ and driven by a dedicated relatively low power amplifier.

\section{Azlmuthal Position Adjustment of Main Ring Bunches}

After bunches are coalesced on Maln Ring flattop at $150 \mathrm{GeV}$ (possibly before for some reason) they will usually have to be moved azimuthally with respect to bunches which have been previously injected into the Tevatron. In order to do this the two systems should first be phase-locked together and the time delay (number of $r f$ periods) between passage of the Maln Ring bunch and the appropriate Tevatron bunch should be measured using signals from directional couplers in each machine. From this information the number of degrees of phase advance required in the Maln Ring can be calculated. The required phase advance can be introduced either by a smooth frequency excursion of the master oscillator or by directly exciting the phase shifters used for counter-phasing of the $r f$. In any case the correction should be done slowly and smoothly. The frequency excursion associated with the required phase advance should be limited to that which keeps the beam within some useful momentum aperture. The frequency range limitation will place a constraint on the time required to do the phase adjustment but it does not appear to be a significant limitation.

Because the identity of the beam phase may become obscured during these processes, it may be reasonable to perform these functions with the beam phase and position feedback loops disabled. This could be accomplished by switching the $\mathrm{rf}$ source to a fixed frequency oscillator at a point beyond the operational phase shifter. The beam bunches and the fixed frequency oscillator must be phase locked together prior to this switch, and in the interest of minimizing the resulting beam disturbance it would seem reasonable to phase lock the oscillator to the beam phase rather than the other way around prior to switching. If the centered beam frequency differs from the target oscillator frequency (because the beam energy and magnet field are not quite correct) the frequency could be allowed to move slowly (with respect to synchrotron period) to the target frequency after the switch but before the counter-phasing operation.

The beam loading compensation voltage must be introduced into the fan-out system at two points following the counter-phasing devices or the beam loading compensation will be counter-phased out just when it is most needed. 